\title{
A NOTE ON DERIVATION PAIRS
}

\section{N. R. NANDAKUMAR}

1. Introduction. Let $G$ be a region in the complex plane and $H(G)$ denote the vector space of functions analytic on $G$. Let $L$ and $M$ be two linear functionals on $H(G)$. The pair $\{L, M\}$ is a derivation pair if

$$
L(f g)=L(f) M(g)+L(g) M(f), \quad f, g \in H(G) .
$$

The purpose of this paper is to determine all derivation pairs generalising a result of L. A. Rubel [1]. This incidentally answers a question raised by him viz., whether the functionals satisfying (1) are continuous.

We denote by $I$ the identity function and $I^{2}$ will then denote the function defined by $I^{2}(z)=I(z)^{2}$. Throughout we assume $\{L, M\}$ to be a derivation pair and $L \neq 0$.

The author is grateful to Professor K. R. Unni for the help and guidance in the preparation of this paper and to Professor Alladi Ramakrishnan, Director of the Institute, for providing good facilities for research. The author is particularly grateful to Professor L. A. Rubel for going through the original manuscript and making valuable suggestions.

ThEOREM. Let $\{L, M\}$ be a derivation pair. Then one of the following is true:

(i) there exists a $z_{1} \in G$ such that

$$
L(f)=L(1) f\left(z_{1}\right), \quad M(f)=\frac{1}{2} f\left(z_{1}\right), \quad f \in H(G) ;
$$

(ii) there exists a $z_{1} \in G$ such that

$$
L(f)=L(I) f^{\prime}\left(z_{1}\right), \quad M(f)=f\left(z_{1}\right), \quad f \in H(G) ;
$$

(iii) there exists $z_{1}, z_{2} \in G\left(z_{1} \neq z_{2}\right)$ such that

$$
L(f)=\frac{L(I)}{z_{1}-z_{2}}\left(f\left(z_{1}\right)-f\left(z_{2}\right)\right), \quad M(f)=\frac{1}{2}\left(f\left(z_{1}\right)+f\left(z_{2}\right)\right), \quad f \in H(G) .
$$

\section{Lemmas.}

Lemma 1. If $N$ is a multiplicative linear functional on $H(G)$, then there exists a $z_{0} \in G$ such that $N(f)=f\left(z_{0}\right), f \in H(G)$.

Received by the editors August 7, 1968. 
The proof of the above lemma is simple and we include it for the sake of completeness.

Proof. Since $N$ is multiplicative we have $N(f)=N(f) N(1)$ which implies $N(1)=1$. Let $N(I)=z_{0}$ so that $N\left(I-z_{0}\right)=N(I)-z_{0} N(1)=0$. We now claim that this $z_{0}$ will satisfy our requirement. First we show that $z_{0} \in G$. Suppose not. Then $1 /\left(I-z_{0}\right) \in H(G)$ and

$$
1=N\left(\left(I-z_{0}\right) \frac{1}{\left(I-z_{0}\right)}\right)=N\left(I-z_{0}\right) N\left(\frac{1}{I-z_{0}}\right)=0
$$

which is impossible.

Now let $f \in H(G)$. Consider the analytic function $g$ defined by $\left(I-z_{0}\right) g=f-f\left(z_{0}\right)$. Applying $N$ to the function $\left(I-z_{0}\right) g$ we obtain $0=N(f)-N\left(f\left(z_{0}\right)\right)$ or $N(f)=f\left(z_{0}\right)$. This completes the proof of lemma.

Lemma 2. If $L(1) \neq 0$, then $L / L(1)$ and $2 M$ are multiplicative.

Proof. By (1) it follows that $L(1)=2 L(1) M(1)$ so that $M(1)=\frac{1}{2}$. Then again using (1) it is easy to show that

$$
M(f)=L(f) / 2 L(1), \quad f \in H(G) .
$$

Substituting this value of $M$ in (1) the result follows.

When $L(1)=0$, we have $L(f)=L(f) M(1)$ and since $L \neq 0$, this implies that $M(1)=1$.

Lemma 3. Let $\{L, M\}$ be a derivation pair and suppose $L(1)=0$. Then

(a) $L(I) \neq 0$.

(b) $M$ is multiplicative when $M\left(I^{2}\right)=M(I)^{2}$.

(c) If $f$ is defined at $z_{0}$ and $f \in H(G)$, then

$$
L(f)=L(I) M\left(\frac{f-f\left(z_{0}\right)}{I-z_{0}}\right), \quad f \in H(G),
$$

where $z_{0}=M(I)$.

Proof. (a) Suppose $L(I)=0$. Let $M(I)=z_{0}$. If $z_{0} \notin G$, and $f \in H(G)$, define $f\left(z_{0}\right)=0$. For all $f \in H(G),\left(f-f\left(z_{0}\right)\right) /\left(I-z_{0}\right) \in H(G)$ so that

$$
\begin{aligned}
L(f) & =L\left(f-f\left(z_{0}\right)\right)=L\left(\left(I-z_{0}\right) \frac{f-f\left(z_{0}\right)}{I-z_{0}}\right) \\
& =L(I) M\left(\frac{f-f\left(z_{0}\right)}{I-z_{0}}\right)=0, \quad f \in H(G) .
\end{aligned}
$$

Hence $L=0$ which is a contradiction. This proves (a). 
(b) Using (1) we obtain on the one hand

$$
L\left(I^{2} f\right)=L\left(I^{2}\right) M(f)+L(f) M\left(I^{2}\right)=2 L(I) M(I) M(f)+L(f) M(I)^{2}
$$

and on the other,

$$
\begin{aligned}
L\left(I^{2} f\right) & =L(I \cdot I f)=L(I) M(I f)+L(I f) M(I) \\
& =L(I) M(I f)+L(I) M(I) M(f)+L(f) M(I)^{2} .
\end{aligned}
$$

Comparing the two expressions for $L\left(I^{2} f\right)$ and noting that $L(I) \neq 0$, we get $M(I f)=M(I) M(f), f \in H(G)$. From this relation it is easy to show (as in Lemma 1 ) that there exists a $z_{0} \in G$ such that $M(f)=f\left(z_{0}\right)$, $f \in H(G)$. This implies $M$ is multiplicative.

(c) $\left(f-f\left(z_{0}\right)\right) /\left(I-z_{0}\right) \in H(G)$ and

$$
\begin{aligned}
L(f) & =L\left(f-f\left(z_{0}\right)\right)=L\left(\left(I-z_{0}\right) \frac{f-f\left(z_{0}\right)}{I-z_{0}}\right) \\
& =L\left(I-z_{0}\right) M\left(\frac{f-f\left(z_{0}\right)}{I-z_{0}}\right)+M\left(I-z_{0}\right) L\left(\frac{f-f\left(z_{0}\right)}{I-z_{0}}\right) \\
& =L(I) M\left(\frac{f-f\left(z_{0}\right)}{I-z_{0}}\right)
\end{aligned}
$$

since $L(1)=0$ and $M\left(I-z_{0}\right)=0$.

This completes the proof of lemma.

Now if $f, g \in H(G)$ and are defined at $z_{0}$, then applying (c) to $L(f g)$, $L(f)$ and $L(g)$ and substituting in (1) we get

$$
\begin{aligned}
M\left(\frac{f g-f\left(z_{0}\right) g\left(z_{0}\right)}{I-z_{0}}\right)= & M(f) M\left(\frac{g-g\left(z_{0}\right)}{I-z_{0}}\right) \\
& +M(g) M\left(\frac{f-f\left(z_{0}\right)}{I-z_{0}}\right) .
\end{aligned}
$$

Put $f=I^{2}-z_{0} I$,

$$
g=1 /\left(I-z_{1}\right) \quad\left(z_{1} \notin G, z_{1} \neq z_{0}\right) .
$$

On noting that $I /\left(I-z_{1}\right)=1+z_{1} /\left(I-z_{1}\right)$, (2) simplifies with these special values to

$$
M\left(\frac{1}{I-z_{1}}\right)\left\{\frac{M\left(I^{2}\right)-z_{0}{ }^{2}}{z_{1}-z_{0}}+z_{0}-z_{1}\right\}=1 .
$$

3. Proof of theorem. Case (i). $L(1) \neq 0$. Then $L / L(1)$ is multiplicative by Lemma 2 . By Lemma 1 , there exists $z_{1} \in G$ such that $L(f) / L(1)$ 
$=f\left(z_{1}\right), f \in H(G)$. Also $M(f)=L(f) / 2 L(1)=f\left(z_{1}\right) / 2, f \in H(G)$. This gives (i) of our theorem.

Case (ii). $L(1)=0$. We have two possibilities $M\left(I^{2}\right)=M(I)^{2}$ or $M\left(I^{2}\right) \neq M(I)^{2}$.

If $M\left(I^{2}\right)=M(I)^{2}$, then $M$ is multiplicative by Lemma 3. Apply Lemma 1 to get $z_{1} \in G$ such that $M(f)=f\left(z_{1}\right), f \in H(G)$. Now we will prove $L(f)=L(I) f^{\prime}\left(z_{1}\right)$. Recall that $M(I)=z_{1}$. Since $z_{1} \in G$, it follows that $\left(f-f\left(z_{1}\right)\right) /\left(I-z_{1}\right) \in H(G)$ for all $f \in H(G)$ and then, by (1) and $L(1)=0$,

$$
\begin{aligned}
L(f)=L\left(f-f\left(z_{1}\right)\right) & =L\left(\left(I-z_{1}\right)\left(f-f\left(z_{1}\right)\right) /\left(I-z_{1}\right)\right) \\
& =L(I) M\left(\left(f-f\left(z_{1}\right)\right) /\left(I-z_{1}\right)\right)=L(I) f^{\prime}\left(z_{1}\right)
\end{aligned}
$$

and we obtain (ii) of our theorem.

It remains to consider the case when $M\left(I^{2}\right) \neq M(I)^{2}=z_{0}^{2}$ and $L(I)=0$.

Since $M\left(I^{2}\right)-z_{0}^{2} \neq 0$, there are two distinct roots, $z_{1}$ and $z_{2}$ say, of

$$
\left(M\left(I^{2}\right)-z_{0}^{2}\right) /\left(z-z_{0}\right)+z_{0}-z=0 .
$$

These roots satisfy

$$
z_{1}+z_{2}=2 z_{0}, \quad z_{1} z_{2}=2 z_{0}^{2}-M\left(I^{2}\right),
$$

so that

(4) $M\left\{\left(I-z_{1}\right)\left(I-z_{2}\right)\right\}=M\left(I^{2}\right)-\left(z_{1}+z_{2}\right) M(I)+z_{1} z_{2} M(1)=0$.

Also by (1), (4) and $L(1)=0$,

(5) $L\left\{\left(I-z_{1}\right)\left(I-z_{2}\right)\right\}=L(I) M\left(I-z_{2}\right)+L(I) M\left(I-z_{1}\right)=0$.

$z_{1} \in G$, since otherwise $M\left(1 /\left(I-z_{1}\right)\right)$ would be finite, contradicting (3). Similarly $z_{2} \in G$.

We can now prove that we have case (iii) of the theorem.

Let $f \in H(G)$. Then

$$
g=\frac{1}{I-z_{2}}\left[\frac{f-f\left(z_{1}\right)}{I-z_{1}}-\frac{f\left(z_{2}\right)-f\left(z_{1}\right)}{z_{2}-z_{1}}\right] \in H(G) .
$$

Applying (1), (4), and (5) to

$$
\left(I-z_{1}\right)\left(I-z_{2}\right) g=\frac{1}{z_{2}-z_{1}}\left[\left(z_{2}-z_{1}\right)\left(f-f\left(z_{1}\right)\right)-\left(I-z_{1}\right)\left(f\left(z_{2}\right)-f\left(z_{1}\right)\right)\right] \text {. }
$$

We obtain 


$$
L(f)=\frac{L(I)}{z_{1}-z_{2}}\left[f\left(z_{1}\right)-f\left(z_{2}\right)\right], \quad f \in H(G) .
$$

From the relation $L\left(\left(I-z_{0}\right) f\right)=L(I) M(f), f \in H(G)$, we obtain $M(f)$ $=\frac{1}{2}\left(f\left(z_{1}\right)+f\left(z_{2}\right)\right), f \in H(G)$. The theorem is completely proved.

\section{REFERENCE}

1. L. A. Rubel, Derivation pairs on the holomorphic functions, Funkcial. Ekvac. 10 (1967), 225-227.

The Institutr. of Mathematical Sciences, Madras, India 